\title{
Reply to the correspondence letter by P. Toma: usefulness of ultrasound findings in bronchiolitis
}

\author{
Vito Antonio Caiulo • Luna Gargani • Silvana Caiulo • \\ Andrea Fisicaro • Fulvio Moramarco • Giuseppe Latini • \\ Eugenio Picano
}

Received: 9 January 2013 / Accepted: 9 January 2013 /Published online: 20 January 2013

(C) Springer-Verlag Berlin Heidelberg 2013

We thank Dr. Tomà for his interest in our article, and we are glad to have the chance to clarify a few more issues.

We agree that hyperinflation is frequent in bronchiolitis. However, laterolateral radiographs are generally more sensitive to detect hyperinflation and, as specified in the paper, we only performed posterior-anterior radiographs and, moreover, with patients in the supine position. It is likely that standard posterior-anterior and laterolateral radiographs would have shown a higher prevalence of hyperinflation.

Dr. Tomà argues that "US cannot reliably differentiate pneumonia from atelectasis". Although this differential diagnosis is not always possible, as it happens also for other imaging tests, some lung ultrasound signs can significantly help to differentiate these two conditions [1, 2, 4], as it is also stated in the recently published "International evidencebased recommendations for point-of-care lung ultrasound" [3].

Dr. Tomà is doubtful about the meaning of the small subpleural lung consolidations and pleural line abnormalities because these aspects can also be related to incomplete expansion of the alveoli, which can be observed in healthy lungs. We agree with that; but in this study, patients were followed-up for more than 10 days, in order to obtain more information on the sonomorphology of the pleuropulmonary

V. A. Caiulo $(\bowtie)$

Italian National School of Pediatric Ultrasound of Italian

Federation of Pediatricians, Brindisi, Italy

e-mail: antoniocaiulo@inwind.it

\section{Gargani $\cdot$ E. Picano}

Institute of Clinical Physiology, National Council of Research,

Division of Pisa, Pisa, Italy

\section{S. Caiulo}

Department of Pediatrics, Vita-Salute San Raffaele University, San Raffaele Scientific Institute, Milan, Italy abnormalities during the course of the disease. In all cases, clinical improvement was associated with the disappearance of lung ultrasound findings. Lung ultrasound has certainly many limitations and many aspects still need to be clarified. Given the high versatility of the technique and the very promising data obtained in some pathological conditions, we believe it is of importance to describe the lung ultrasound patterns of specific disease, even when, as in bronchiolitis, the diagnosis is mainly based on the clinical signs and symptoms.

\section{References}

1. Lichtenstein D, Meziere G, Seitz J (2009) The dynamic air bronchogram. A lung ultrasound sign of alveolar consolidation ruling out atelectasis. Chest 135:1421-1425

2. Mathis G (2008) Chest sonography. Springer, Heidelberg

3. Volpicelli G, Elbarbary M, Blaivas M, Lichtenstein DA, Mathis G, Kirkpatrick AW et al (2012) International evidence-based recommendations for point-of-care lung ultrasound. Intensive Care Med 38:577-591

4. Weinberg B, Diakoumakis EE, Kass EG, Seife B, Zvi ZB (1986) The air bronchogram: sonographic demonstration. AJR Am J Roentgenol 147:593-595 